\title{
Murine pluripotent stem cells that escape differentiation inside teratomas maintain pluripotency
}

\author{
Yangli Pei ${ }^{1,2}$, Liang Yue ${ }^{2}$, Wei Zhang ${ }^{2}$, Jinzhu Xiang ${ }^{2}{ }^{2}$ Zhu Ma ${ }^{3}$, Jianyong Han ${ }^{\text {Corresp. } 2}$ \\ ${ }^{1}$ State Key Laboratory of Animal Nutrition, Institute of Animal Sciences, Chinese Academy of Agricultural Sciences, Beijing, China \\ 2 State Key Laboratories for Agrobiotechnology, College of Biological Sciences, China Agricultural University, Beijing, China \\ 3 Beijing Dairy Cattle Center, Beijing, China \\ Corresponding Author: Jianyong Han \\ Email address: hanjy@cau.edu.cn
}

Background. Pluripotent stem cells (PSCs) offer immense potential as a source for regenerative therapies. The teratoma assay is widely used in the field of stem cells and regenerative medicine, but the cell composition of teratoma is still elusive.

Methods. We utilized PSCs expressing enhanced green fluorescent protein (EGFP) under the control of the Pou5f1 promoter to study the persistence of potential pluripotent cells during teratoma formation in vivo. OCT4-MES (mouse embryonic stem cells) were isolated from the blastocysts of 3.5-day OCT4-EGFP mice (transgenic mice express EGFP cDNA under the control of the Pou5f1 promoter) embryos, and TG iPS 1-7 (induced pluripotent stem cells) were generated from mouse embryonic fibroblasts (MEFs) from 13.5-day OCT4-EGFP mice embryos by infecting them with a virus carrying OCT4, SOX2, KLF4 and C-MYC. These pluripotent cells were characterized according to their morphology and expression of pluripotency markers. Their differentiation ability was studied with in vivo teratoma formation assays. Further differences between pluripotent cells were examined by real-time quantitative PCR (qPCR).

Results. The results showed that s everal OCT4-expressing PSCs escaped differentiation inside of teratomas, and these escaped cells (MES-FT, GFP-positive cells separated from OCT4-MES-derived teratomas; and iPS-FT, GFP-positive cells obtained from teratomas formed by TG iPS 1-7) retained their pluripotency. Interestingly, a small number of GFP-positive cells in teratomas formed by MES-FT and iPSFT ( MES-ST, GFP-positive cells isolated from MES-FT-derived teratomas; iPS-ST, GFP-positive cells obtained from teratomas formed by iPS-FT ) were still pluripotent, as shown by alkaline phosphatase (AP) staining, immunofluorescent staining and PCR. MES-FT, iPS-FT, MES-ST and iPS-ST cells also expressed several markers associated with germ cell formation, such as Dazl, Stella and Stra8.

Conclusions. In summary, a small number of PSCs escaped differentiation inside of teratomas, and these cells maintained pluripotency and partially developed towards germ cells. Both escaped PSCs and germ cells present a risk of tumor formation. Therefore, medical workers must be careful in preventing tumor formation when stem cells are used to treat specific diseases. 


\section{pluripotency}

$4{ }^{1}$ State Key Laboratory of Animal Nutrition, Institute of Animal Sciences, Chinese Academy of

5 Agricultural Sciences, Beijing, 100193, P.R. China

6 2State Key Laboratories for Agrobiotechnology, College of Biological Sciences, China

7 Agricultural University, Beijing, 100193, China

$8 \quad{ }^{3}$ Beijing Dairy Cattle Center, Beijing 100193, China

$9 \quad *$ Correspondence: hanjy@cau.edu.cn (JH) 


\section{Abstract}

12 Background. Pluripotent stem cells (PSCs) offer immense potential as a source for regenerative therapies. The teratoma assay is widely used in the field of stem cells and regenerative medicine, but the cell composition of teratoma is still elusive.

Methods. We utilized PSCs expressing enhanced green fluorescent protein (EGFP) under the control of the Pou5fl promoter to study the persistence of potential pluripotent cells during teratoma formation in vivo. OCT4-MES (mouse embryonic stem cells) were isolated from the blastocysts of 3.5-day OCT4-EGFP mice (transgenic mice express EGFP cDNA under the control of the Pou5f1 promoter) embryos, and TG iPS 1-7 (induced pluripotent stem cells) were generated from mouse embryonic fibroblasts (MEFs) from 13.5-day OCT4-EGFP mice embryos by infecting them with a virus carrying OCT4, SOX2, KLF4 and c-MYC. These pluripotent cells were characterized according to their morphology and expression of pluripotency markers. Their differentiation ability was studied with in vivo teratoma formation assays. Further differences between pluripotent cells were examined by real-time quantitative PCR (qPCR).

Results. The results showed that several OCT4-expressing PSCs escaped differentiation inside of teratomas, and these escaped cells (MES-FT, GFP-positive cells separated from OCT4-MESderived teratomas; and iPS-FT, GFP-positive cells obtained from teratomas formed by TG iPS 17) retained their pluripotency. Interestingly, a small number of GFP-positive cells in teratomas formed by MES-FT and iPS-FT (MES-ST, GFP-positive cells isolated from MES-FT-derived teratomas; iPS-ST, GFP-positive cells obtained from teratomas formed by iPS-FT) were still pluripotent, as shown by alkaline phosphatase (AP) staining, immunofluorescent staining and 
32 PCR. MES-FT, iPS-FT, MES-ST and iPS-ST cells also expressed several markers associated with

33 germ cell formation, such as Dazl, Stella and Stra8.

34 Conclusions. In summary, a small number of PSCs escaped differentiation inside of teratomas,

35 and these cells maintained pluripotency and partially developed towards germ cells. Both escaped

36 PSCs and germ cells present a risk of tumor formation. Therefore, medical workers must be careful

37 in preventing tumor formation when stem cells are used to treat specific diseases. 
Introduction

40 Pluripotent stem cells (PSCs), including embryonic stem cells (ESCs) and induced pluripotent

41 stem cells (iPSCs), have the potential to differentiate into all cell types of the body in vitro through

embryoid body formation or in vivo through teratoma formation. Due to these characteristics, stem

cells provide an option for treating a multitude of clinical problems, such as myocardium damage

after heart infarction, spinal cord damage after mechanical injury, brain damage after stroke, age-

related macular degeneration of the retina, liver damage, extensive skin burns, Parkinson's disease,

and diabetes (Abdelalim et al., 2014; Lodi et al., 2011; Orlic et al., 2001; Ratajczak et al., 2016).

When transplanted into immune-compromised mice, undifferentiated PSCs can form teratomas,

consisting of multiple tissue types derived from all three germ layers (Przyborski, 2005;

Takahashiand Yamanaka, 2006). As such, there have been many efforts to differentiate of

pluripotent cells to cells with medical applications in vivo developmental environment. For

example, neural stem cells (NSCs) have been differentiated in vivo through teratoma formation,

and pure NSC populations exhibit properties similar to those of brain-derived NSCs (Hong et al.,

2016). Similarly, fully functional and engraftable hematopoietic stem/progenitor cells (HSPCs),

along with functional myeloid and lymphoid cells, have been isolated from teratomas when human

iPSCs were transplanted into immunodeficient mice (Amabile et al., 2013; Suzuki et al., 2013). In

addition, the teratoma assay can be applied to assess the safety of human PSC-derived cell

populations that are used for therapeutic application since a small number of undifferentiated cells contaminating a given transplant material can be efficiently detected by their multi-lineage differentiation ability (Stachelscheid et al., 2013). 
60 However, the intrinsic self-renewal and pluripotency qualities of PSCs that make them

61 therapeutically promising are responsible for an equally fundamental tumorigenic risk (Lee et al.,

62 2013). Studies on teratomas will contribute to a better understanding of their stepwise development

63 processes and underlying molecular mechanisms and may provide helpful information for the

64 development of tissue engineering technologies (Aleckovicand Simon, 2008). These facts

65 prompted us to address the additional characteristics of teratoma growth and differentiation after

66 PSCs injection.

67 In the present study, we aimed to isolate OCT4-expressing cells that escaped differentiation inside

68 of growing teratomas and to determine whether OCT4-expressing cells still possess self-renewal

69 and pluripotency abilities.

70 Materials \& methods

71 All animal experiments were approved by the Animal Care and Use Committees of the State Key

72 Laboratories for Agrobiotechnology, College of Biological Sciences, China Agricultural

73 University (Approval number: SKLAB-2016-05-01). Briefly, mice were bred in a 12/12 h

74 light/dark period and sacrificed by cervical vertebra dislocation.

\section{Mouse strains}

OCT4-GFP transgenic mice (Model Animal Research Center of Nanjing University) express EGFP (enhanced green fluorescence protein) cDNA under the control of the Pou5f1 promoter, which is active in pluripotent stem cells. This strain is useful for isolating pluripotent stem cells, as they specifically express green fluorescent protein. These OCT4-GFP transgenic mice were the source of the OCT4-MES and OG2 MEFs (mouse embryonic fibroblasts of 13.5-day OCT4-EGFP 
81 mice embryos) used in this study.

\section{Derivation of MES and generation of iPSCs}

To obtain OCT4-MES, uteri containing E3.5 embryos were isolated from timed pregnancies and transferred individually to the wells of a 24-well plate with irradiated mouse embryonic fibroblast (MEF) feeders. After 5 days of incubation, embryo outgrowths were separated from trophectoderm, individually picked, and expanded in MES medium (Dulbecco's modified eagle medium (DMEM) supplemented with 15\% fetal bovine serum (FBS), L-glutamine, nonessential amino acids, $\beta$-mercaptoethanol, and 1,000 U/ml leukemia inhibitory factor).

OG2 MEFs were cultured in MEF medium (DMEM supplemented with 10\% FBS, L-glutamine and nonessential amino acids); infected with retroviruses generated from pMX retroviral vectors encoding mouse Pou5f1, Sox2, Klf4 and $c-M y c$; and cultured on irradiated MEF feeder cells in MES medium. Subsequently, a single ESC-like colony was individually picked and expanded on feeders to establish stable lines. Both OCT4-MES and iPSCs originated from male embryos. Additional details can be found in our previous study (Pei et al., 2015).

\section{Immunofluorescence}

Cells were fixed with $4 \%$ paraformaldehyde, permeabilized with $0.1 \%$ Triton X-100, and blocked with $2 \%$ BSA. The cells were then stained with primary antibodies against OCT4 (Abcam, ab19857, 1:500), SOX2 (Abcam, ab97959, 1:1000), NANOG (Abcam, ab80892, 1:500) and SSEA1 (Abcam, ab16285, 1:200), followed by staining with the respective secondary antibodies conjugated to Alexa Fluor (Invitrogen, A-11008, A-11037, A-21044, 1:1000). Finally, cells were counterstained with DAPI (Sigma, D9542). 
102

103

104

105

106

107

108

109

110

111

112

113

114

115

116

117

118

119

120

121

\section{RNA purification and CDNA preparation}

Feeders were removed by plating ESCs on a gelatin-coated dish for $30 \mathrm{~min}$, and unattached cells were collected by centrifugation. Total RNA was extracted from pure PSCs using Trizol reagent according to the manufacturer's instructions (Invitrogen). RNA was reverse-transcribed using oligo-dT and M-MLV Reverse Transcriptase (Promega, Madison, WI).

\section{Real-time quantitative PCR}

qPCR was performed on a LightCycler 480 II Real-Time PCR System (Roche, Basel, Switzerland) using the LightCycler 480 SYBR Green I Master Mix (Roche, Basel, Switzerland, 4887352001). The qPCR data was analyzed using the comparative CT $\left(2^{-\Delta \Delta C T}\right)$ method as the description by Livak and Schmittgen (Livakand Schmittgen, 2001). The $\Delta$ CT was calculated using Gapdh, EF1 $\alpha$ and $\beta$-tubulin as internal control.

The primers used for qPCR and PCR are listed in table 1.

\section{Teratoma production and analysis}

Approximately $1 \times 10^{6}$ PSCs were suspended in $150 \mu 1$ of PBS (phosphate buffered solution) and subcutaneously injected into the hind limb of NOD/SCID mice to form teratomas. Three weeks after injection, the teratomas were harvested, fixed overnight with $4 \%$ paraformaldehyde, embedded in paraffin, sectioned, HE stained or immunostained (primary antibodies against GFP, Cell Signaling Technology, Cat. 2956, 1: 200; Biotin-Streptavidin horseradish peroxidase detection kit, Beijing zhongshan golden bridge biotechnology company, Cat. SP-9001), and analyzed. 
122

123

124

125

126

127

128

129

130

131

132

133

134

135

136

137

\section{Statistical analysis}

All results are presented as the mean \pm standard deviation. Results were statistically analyzed using SAS (Statistics Analysis System) program. Significance of differences between samples was determined (at the significance level $\mathrm{p}<0.05$ ) using Kruskal-Wallis test.

\section{Results}

\section{Both OCT4-MES and TG iPS 1-7 are pluripotent}

OCT4-EGFP mice express green fluorescent protein under the control of the pluripotencyassociated Pou5f1 promoter and are widely used to study the function of PSCs (Pei et al., 2015). These mice were used to generate mouse embryonic stem cells (MES) and iPSCs. OCT4-MES were isolated from the blastocysts of 3.5-day OCT4-EGFP mice embryos, while other mice were selected to prepare MEFs after day 13.5. The isolated MEFs were used to generate iPSCs by infecting them with a virus carrying OCT4, SOX2, KLF4 and c-MYC. Then, TG iPS 1-7 was selected from the isolated iPSC clones.

Both OCT4-MES and TG iPS 1-7 were maintained on feeder cells in the presence of leukemia inhibitory factor. They both exhibited typical MES-like morphologies (Figure 1a and b). Immunofluorescent staining confirmed the expression of the three master transcription factors (OCT4, NANOG and SOX2) as well as ESC-specific surface marker SSEA-1 in OCT4-MES and TG iPS 1-7 (Figure 1c-j). The PCR results further demonstrated that these cells expressed pluripotency marker genes, including Pou5f1, Sox2, Nanog, Rex1, Tbx3, Nr5a2, Utf1 and Lin28a (Figure 2). Next, in vivo teratoma formation assays were performed to further validate the 
142 pluripotency of OCT4-MES and TG iPS 1-7. Approximately $1 \times 10^{6}$ PSCs were suspended in 150

$143 \mu \mathrm{l}$ of PBS and injected into non-obese diabetic/severe combined immunodeficient (NOD/SCID)

144 mice to form teratomas. Three weeks after injection, OCT4-MES and TG iPS 1-7 formed

145 teratomas in vivo, and hematoxylin and eosin (H\&E) staining confirmed the formation of all three

146 germ layers in each teratoma (Figure 3a-f). These results revealed that OCT4-MES and TG iPS

147 1-7 were pluripotent. Interestingly, we observed OCT4-positive cells growing in clusters in the

148 teratoma masses formed by OCT4-MES and TG iPS 1-7 (Figure 3g, h).

149 OCT4-positive cells from OCT4-MES and TG iPS 1-7 teratomas have self-renewal and

\section{pluripotency qualities}

To quantify the fraction of OCT4-positive pluripotent cells in teratomas generated by OCT4-MES

and TG iPS 1-7, we cut the teratomas into pieces and digested them with trypsin and then cultured

the cells in MEF medium. Three days later, we found that most of these cells separated from

OCT4-MES and that TG iPS 1-7-derived teratomas had the morphology of mouse embryonic cells,

but a small number of cells were round and expressed GFP (Figure 4a, b, d and e). After picking

these cells and culturing them in MES medium, we found that they had typical MES-like

morphologies, and they were AP-positive (Figure 4c, f). We named these cells MES-FT and iPS-

FT, which were derived from OCT4-MES and TG iPS 1-7, respectively. OCT4-expressing MES-

FT and iPS-FT cells were grown in the presence of leukemia inhibitory factor, and they expressed

161 (Figure 2). The immunostaining results showed that these colonies were positive for OCT4,

NANOG, SOX2 and SSEA-1 (Figure 5a-h). We performed in vivo teratoma formation assays to 

in vivo, and the hematoxylin and eosin $(\mathrm{H} \& \mathrm{E})$ staining results confirmed the formation of all three germ layers in each teratoma (Figure 5i-n). As in the results described above, there were also OCT4-positive pluripotent cells in the teratomas formed by MES-FT and iPS-FT (Figure 5o, p).

\section{OCT4-positive cells from MES-FT and iPS-FT teratomas are still pluripotent}

We discovered several round and bright cells expressing OCT4-GFP under a microscope in cells separated from teratomas formed by MES-FT and iPS-FT cells (Figure 6a, b d and e). These round and bright cells formed AP positive clones (Figure 6c, f). We named these cells MES-ST and iPSST. OCT4-expressing MES-ST and iPS-ST cells also expressed pluripotency marker genes, including Pou5f1, Sox2, Nanog, Rex1, Tbx3, Nr5a2, Utf1 and Lin28a (Figure 2), and the immunostaining results demonstrated that they expressed the stemness regulators OCT4, NANOG, SOX2 and SSEA-1 (Figure 6g-n).

The above results showed that OCT4-MES, TG iPS 1-7, MES-ST, iPS-ST, MES-ST and iPS-ST

had pluripotency characteristics. However, MES-FT, iPS-FT, MES-ST and iPS-ST were survivors

of the differentiation environment, so we wanted to know whether there were differences among

these cells. Thus, we next investigated their differences.

\section{OCT4-positive cells separated from teratomas express germ cell marker genes}

To explore the gene expression patterns of OCT4-MES, TG iPS 1-7, MES-FT, iPS-FT, MES-ST and iPS-ST, cDNA was prepared from these cells without feeders for gene expression analysis.

First, we detected the expression of pluripotency genes. When normalized to the values for OCT4-

MES cells, the expression level of Pou5f1 was higher in MES-FT, and that of Lin28a was higher 
184

185

186

in both MES-FT and MES-ST cells, but there were no differences in the Nanog expression levels between these three cell lines (Figure 7a). When normalized to the values for TG iPS 1-7 cells, iPS-FT and iPS-ST both highly expressed Pou5f1 and Nanog (Figure 7b). However, there were no differences in the expression level of $\operatorname{Lin} 28 a$ (Figure 7b). The expression of pluripotency marker genes in these cells varied slightly, but they were all within reasonable levels. Thus, these cell types were all pluripotent.

Previous results have shown that PSCs that escape from differentiation inside of embryonic bodies express several markers associated with germ cell formation (Attia et al., 2014). As such, we further assayed the differences between the expression levels of important germ cell-specific genes (Dazl, Stella, Stra8, Vasa) in MES-FT, iPS-FT, MES-ST and iPS-ST (Figure 7c, d). When normalized to the values for OCT4-MES, Dazl and Stella were more highly expressed in MES-FT cells, and the expression level of Stra8 was elevated nine-fold and ten-fold in MES-FT and MESST, respectively.

Similarly, iPS-FT and iPS-ST highly expressed Dazl, Stra8 and Vasa than TG iPS 1-7. iPS-FT also highly expressed Stella. The above results show that OCT4-positive cells separated from teratomas have elevated expression of several markers associated with germ cell formation, such as Dazl, Stella and Stra8.

\section{Discussion}

ESCs and iPSCs are characterized by their ability to develop into any cell type of the adult organism. As such, they can be widely applied to the treatment of many diseases. This is especially true for iPSCs, as they do not present ethical issues. 
205 A previous report demonstrated the presence of undifferentiated human ESCs expressing the 206 surface marker CD133 (Ritnerand Bernstein, 2010). However, no additional research has been 207 performed to investigate the characteristics of those undifferentiated cells in teratomas. Therefore, 208 in this study, we isolated OCT4-GFP positive cells, MES-FT and iPS-FT, from teratomas 209 generated by OCT4-MES and TG iPS 1-7, respectively. MES-FT and iPS-FT exhibit MES-like morphologies, express pluripotency marker genes and proteins, and can generate all three germ layers in an in vivo differentiation model. We discovered that there were still pluripotent cells in the teratomas formed by MES-FT and iPS-FT, so we separated them from the teratoma mass and named them MES-ST and iPS-ST. Further study confirmed that these isolated cells (MES-ST and iPS-ST) retained pluripotency and were capable of differentiation. From these results, it can be inferred that a subset of PSCs escape differentiation during in vivo differentiation, and the escaped cells retain their PSC characteristics in the appropriate environment. Since the escaped PSCs (MES-FT, iPS-FT, MES-ST and iPS-ST) still possessed PSC-like characteristics, these cells may progress to tumor formation at an undefined later time point.

Bottai et al. reported that they used $5 \times 10^{5}$ undifferentiated murine ESCs to cure spinal cord injury.

However, some of the transplanted ESCs were found as dense aggregates in the tissue (Bottai et al., 2010). This result supports our view that ESCs can be maintained in vivo. Another study showed that transplantation of $1-2 \times 10^{6}$ MES cells into SV129 mice led to tumor formation in $100 \%$ of cases, whereas transplantation of $5 \times 10^{5}$ cells produced tumors in 2 of 6 mice and transplantation of $1 \times 10^{5}$ ESCs gave rise to tumor formation in 1 of 6 transplanted mice within 100 days (Dressel et al., 2008). It can be deduced that there is likely a niche within teratomas that nurse 
226

227

228

229

230

231

232

233

234

235

236

237

238

239

240

241

242

243

244

245

246

PSCs, and the number of cells determines the niche environment. The more PSCs used for transplantation, the higher probability of tumor formation.

The escaped PSCs (MES-FT, iPS-FT, MES-ST and iPS-ST) showed slight similarities to primordial germ cells (PGCs), as shown by the high expression of Pou5f1, Dazl, Stella and Stra8 in MES-FT, MES-ST, iPS-FT, and iPS-ST. Pou5f1, Dazl, Stella, Stra8 and Vasa are well-known germ cell markers, and they are also commonly expressed in ESCs (Cauffman et al., 2005; Kehler et al., 2004; Tedesco et al., 2009; Toyooka et al., 2000; Wongtrakoongate et al., 2013). Stra8 is required for the chromosomal program of meiotic prophase (Soh et al., 2015). Dazl, an intrinsic meiotic competence factor, is required for Stra8-mediated initiation of meiosis in germ cells (Lin et al., 2008). Overexpression of Stra8 and Dazl genes promotes the transdifferentiation of mesenchymal stem cells and ESCs in vitro toward PGCs (Li et al., 2017; Shi et al., 2014). The elevated expression of Pou5f1, Dazl, Stella and Stra8 might indicate that the GFP positive cells separated from teratomas partially develop towards germ cells. This suggests that it is possible to isolate PGCs from teratoma differentiation models.

\section{Conclusions}

In summary, we found a small number of OCT4-expressing PSCs that escaped differentiation inside teratomas. The escaped cells kept their unique properties of self-renewal and pluripotency and were able to form teratomas in vivo. They also expressed several markers associated with germ cell formation, such as Pou5f1, Dazl, Stella and Stra8, suggesting that these cells may partially differentiate into germ cells. Therefore, this study serves as a warning that medical workers using stem cells to treat specific diseases must pay careful attention to prevent tumor formation because 
247 OCT4-expressing cells retain pluripotency, and it is feasible to isolate germ cells from teratomas.

248 This study of PSCs that remain undifferentiated within teratomas has provided critical information

249 for further investigation of the applications of stem cell therapy and for obtaining germ cells from 250 in vivo differentiation models. 
252

253

254

255

256

257

258

259

260

261

262

263

264

265

266

267

268

269

270

271

272

273

274

275

276

277

278

279

280

281

282

283

284

285

286

287

288

289

290

291

\section{References}

Abdelalim, E.M., Bonnefond, A., Bennaceur-Griscelli, A.,Froguel, P. (2014). Pluripotent stem cells as a potential tool for disease modelling and cell therapy in diabetes. Stem cell reviews 10, 327-337.

Aleckovic, M.,Simon, C. (2008). Is teratoma formation in stem cell research a characterization tool or a window to developmental biology? Reproductive biomedicine online 17, 270-280.

Amabile, G., Welner, R.S., Nombela-Arrieta, C., D'Alise, A.M., Di Ruscio, A., Ebralidze, A.K., Kraytsberg, Y., Ye, M., Kocher, O., Neuberg, D.S., Khrapko, K., Silberstein, L.E.,Tenen, D.G. (2013). In vivo generation of transplantable human hematopoietic cells from induced pluripotent stem cells. Blood 121, 1255-1264.

Attia, W.A., Abd El Aziz, O.M., Spitkovsky, D., Gaspar, J.A., Droge, P., Suhr, F., Sabour, D., Winkler, J., Meganathan, K., Jagtap, S., Khalil, M., Hescheler, J., Konrad, B., Agapios, S.,Kurt, P. (2014). Evidence for selfmaintaining pluripotent murine stem cells in embryoid bodies. Stem cell reviews 10, 1-15.

Bottai, D., Cigognini, D., Madaschi, L., Adami, R., Nicora, E., Menarini, M., Di Giulio, A.M.,Gorio, A. (2010). Embryonic stem cells promote motor recovery and affect inflammatory cell infiltration in spinal cord injured mice. Experimental Neurology 223, 452-463.

Cauffman, G., Van de Velde, H., Liebaers, I.,Van Steirteghem, A. (2005). DAZL expression in human oocytes, preimplantation embryos and embryonic stem cells. Molecular human reproduction 11, 405-411.

Dressel, R., Schindehütte, J., Kuhlmann, T., Elsner, L., Novota, P., Baier, P.C., Schillert, A., Bickeböller, H., Herrmann, T., Trenkwalder, C., Paulus, W.,Mansouri, A. (2008). The tumorigenicity of mouse embryonic stem cells and in vitro differentiated neuronal cells is controlled by the recipients' immune response. In PLoS One, pp. e2622. Hong, Y.J., Kim, J.S., Choi, H.W., Song, H., Park, C.,Do, J.T. (2016). In Vivo Generation of Neural Stem Cells Through Teratoma Formation. Stem cells and development 25, 1311-1317.

Kehler, J., Tolkunova, E., Koschorz, B., Pesce, M., Gentile, L., Boiani, M., Lomeli, H., Nagy, A., McLaughlin, K.J., Scholer, H.R.,Tomilin, A. (2004). Oct4 is required for primordial germ cell survival. EMBO reports 5, 1078-1083.

Lee, A.S., Tang, C., Rao, M.S., Weissman, I.L.,Wu, J.C. (2013). Tumorigenicity as a clinical hurdle for pluripotent stem cell therapies. Nature medicine 19, 998-1004.

Li, P.Z., Yan, G.Y., Han, L., Pang, J., Zhong, B.S., Zhang, G.M., Wang, F.,Zhang, Y.L. (2017). Overexpression of STRA8, BOULE, and DAZL Genes Promotes Goat Bone Marrow-Derived Mesenchymal Stem Cells In Vitro Transdifferentiation Toward Putative Male Germ Cells. Reproductive sciences (Thousand Oaks, Calif) 24, 300-312. Lin, Y., Gill, M.E., Koubova, J.,Page, D.C. (2008). Germ cell-intrinsic and -extrinsic factors govern meiotic initiation in mouse embryos. Science (New York, NY) 322, 1685-1687.

Livak, K.J.,Schmittgen, T.D. (2001). Analysis of relative gene expression data using real-time quantitative PCR and the 2(-Delta Delta C(T)) Method. Methods (San Diego, Calif) 25, 402-408.

Lodi, D., Iannitti, T.,Palmieri, B. (2011). Stem cells in clinical practice: applications and warnings. Journal of experimental \& clinical cancer research : CR 30, 9.

Orlic, D., Kajstura, J., Chimenti, S., Jakoniuk, I., Anderson, S.M., Li, B., Pickel, J., McKay, R., Nadal-Ginard, B., Bodine, D.M., Leri, A.,Anversa, P. (2001). Bone marrow cells regenerate infarcted myocardium. Nature 410, 701705.

Pei, Y., Yue, L., Zhang, W., Wang, Y., Wen, B., Zhong, L., Xiang, J., Li, J., Zhang, S., Wang, H., Mu, H., Wei, Q.,Han, J. (2015). Improvement in Mouse iPSC Induction by Rab32 Reveals the Importance of Lipid Metabolism during Reprogramming. Scientific reports 5, 16539. 
292

293

294

295

296

297

298

299

300

301

302

303

304

305

306

307

308

309

310

311

312

313

314

315

316

317

318

319

320

321

Przyborski, S.A. (2005). Differentiation of human embryonic stem cells after transplantation in immune-deficient mice. Stem cells (Dayton, Ohio) 23, 1242-1250.

Ratajczak, M.Z., Bujko, K.,Wojakowski, W. (2016). Stem cells and clinical practice: new advances and challenges at the time of emerging problems with induced pluripotent stem cell therapies. Polskie Archiwum Medycyny Wewnetrznej 126, 879-890.

Ritner, C.,Bernstein, H.S. (2010). Fate Mapping of Human Embryonic Stem Cells by Teratoma Formation. J Vis Exp 42, e2036.

Shi, Q.Q., Sun, M., Zhang, Z.T., Zhang, Y.N., Elsayed, A.K., Zhang, L., Huang, X.M.,Li, B.C. (2014). A screen of suitable inducers for germline differentiation of chicken embryonic stem cells. Animal reproduction science 147, 7485.

Soh, Y.Q., Junker, J.P., Gill, M.E., Mueller, J.L., van Oudenaarden, A.,Page, D.C. (2015). A Gene Regulatory Program for Meiotic Prophase in the Fetal Ovary. PLoS genetics 11, e1005531.

Stachelscheid, H., Wulf-Goldenberg, A., Eckert, K., Jensen, J., Edsbagge, J., Bjorquist, P., Rivero, M., Strehl, R., Jozefczuk, J., Prigione, A., Adjaye, J., Urbaniak, T., Bussmann, P., Zeilinger, K.,Gerlach, J.C. (2013). Teratoma formation of human embryonic stem cells in three-dimensional perfusion culture bioreactors. Journal of tissue engineering and regenerative medicine 7, 729-741.

Suzuki, N., Yamazaki, S., Yamaguchi, T., Okabe, M., Masaki, H., Takaki, S., Otsu, M.,Nakauchi, H. (2013). Generation of engraftable hematopoietic stem cells from induced pluripotent stem cells by way of teratoma formation. Molecular therapy : the journal of the American Society of Gene Therapy 21, 1424-1431.

Takahashi, K.,Yamanaka, S. (2006). Induction of pluripotent stem cells from mouse embryonic and adult fibroblast cultures by defined factors. Cell 126, 663-676.

Tedesco, M., La Sala, G., Barbagallo, F., De Felici, M.,Farini, D. (2009). STRA8 shuttles between nucleus and cytoplasm and displays transcriptional activity. The Journal of biological chemistry 284, 35781-35793.

Toyooka, Y., Tsunekawa, N., Takahashi, Y., Matsui, Y., Satoh, M.,Noce, T. (2000). Expression and intracellular localization of mouse Vasa-homologue protein during germ cell development. Mechanisms of development 93, 139149.

Wongtrakoongate, P., Jones, M., Gokhale, P.J.,Andrews, P.W. (2013). STELLA facilitates differentiation of germ cell and endodermal lineages of human embryonic stem cells. PLoS One 8 , e56893. 


\section{Table 1 (on next page)}

Sequence of primers used in this study. 


\begin{tabular}{|c|c|c|}
\hline Gene & & Sequence $\left(5^{\prime}-3^{\prime}\right)$ \\
\hline \multirow[t]{2}{*}{ Gapdh } & Forward & AGGTCGGTGTGAACGGATTTG \\
\hline & Reverse & TGTAGACCATGTAGTTGAGGTCA \\
\hline \multirow[t]{2}{*}{$\beta$-tubulin } & Forward & TGAGGCCTCCTCTCACAAGTA \\
\hline & Reverse & CCGCACGACATCTAGGACTG \\
\hline \multirow[t]{2}{*}{ EF $1 \alpha$} & Forward & GTGTTGTGAAAACCACCGCT \\
\hline & Reverse & AGGAGCCCTTTCCCATCTCA \\
\hline \multirow[t]{2}{*}{ Pou5f1 } & Forward & GTTGGAGAAGGTGGAACCAA \\
\hline & Reverse & СТCCTTCTGCAGGGCTTTC \\
\hline \multirow[t]{2}{*}{ Sox 2} & Forward & AAGGGTTCTTGCTGGGTTTT \\
\hline & Reverse & AGACCACGAAAACGGTCTTG \\
\hline \multirow[t]{2}{*}{ Utf1 } & Forward & GTCCGGACCCTTCGATAACC \\
\hline & Reverse & CTCGGCCTCTTGCTCCAC \\
\hline \multirow[t]{2}{*}{ Nanog } & Forward & TTCTTGCTTACAAGGGTCTGC \\
\hline & Reverse & AGAGGAAGGGCGAGGAGA \\
\hline \multirow[t]{2}{*}{ Rex1 } & Forward & CAGTTCGTCCATCTAAAAAGGGAGG \\
\hline & Reverse & TCTTAGCTGCTTCCTTGAACAATGCC \\
\hline \multirow[t]{2}{*}{ Tbx3 } & Forward & ATCGCCGTTACTGCCTATCA \\
\hline & Reverse & TGCAGTGTGAGCTGCTTTCT \\
\hline \multirow[t]{2}{*}{$\operatorname{Lin} 28 \mathrm{a}$} & Forward & GTCTTTGTGCACCAGAGCAAG \\
\hline & Reverse & ATGGATTCCAGACCCTTGGC \\
\hline \multirow[t]{2}{*}{$\mathrm{Nr} 5 \mathrm{a} 2$} & Forward & TAGGACCGGAAAGCGTCTGC \\
\hline & Reverse & GCTTCCGTCTCCACTTTGGG \\
\hline \multirow[t]{2}{*}{ Dazl } & Forward & GCCCGCAAAAGAAGTCTGTG \\
\hline & Reverse & ACCAACAACCCCCTGAGATG \\
\hline \multirow[t]{2}{*}{ Stella } & Forward & GAGAAGACTTGTTCGGATTGAGC \\
\hline & Reverse & CATCGTCGACAGCCAGGG \\
\hline
\end{tabular}




$\begin{array}{ccc}\text { Stra8 } & \text { Forward } & \text { CTCCTCCTCCACTCTGTTGC } \\ & \text { Reverse } & \text { GCGGCAGAGACAATAGGAAG } \\ \text { Vasa } & \text { Forward } & \text { ACCAAGATCAGGGGACACAG } \\ & \text { Reverse } & \text { TAACCACCTCGACCACTTCC }\end{array}$

1 


\section{Figure 1}

OCT4-MES and TG iPS 1-7 are pluripotent.

( $a$ and b) Phase contrast images of OCT4-MES (a) and TG iPS 1-7 (b). Both types of cells exhibit typical MESlike morphologies.

(c-j) Immunofluorescent staining showing that both OCT4-MES (c-f) and TG IPS 1-7 (g-j) express the pluripotency markers OCT4 (c and g), NANOG ( $d$ and $h$ ), SOX2 (e and i) and SSEAl( $f$ and $j$ ).

a

OCT4MES

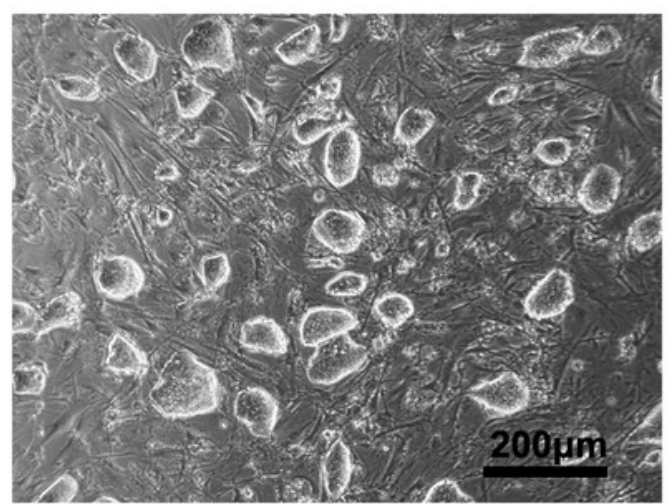

C

OCT4-

MES

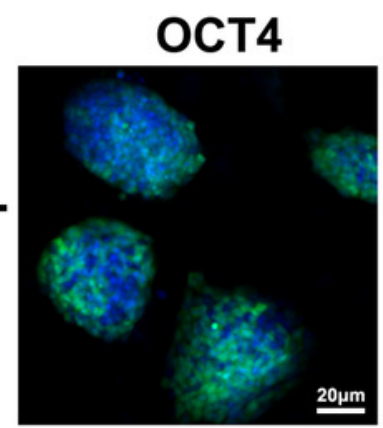

d

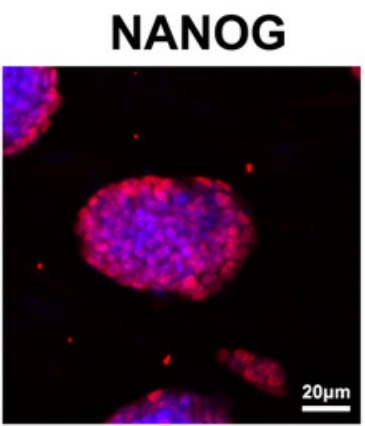

g

TG iPS

1-7
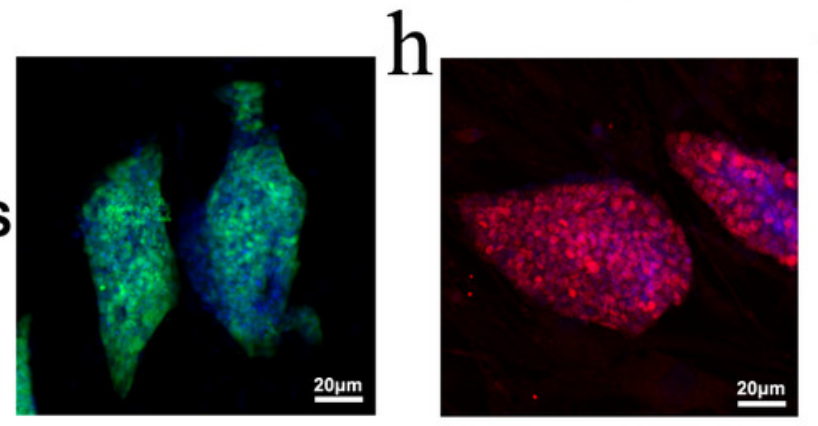
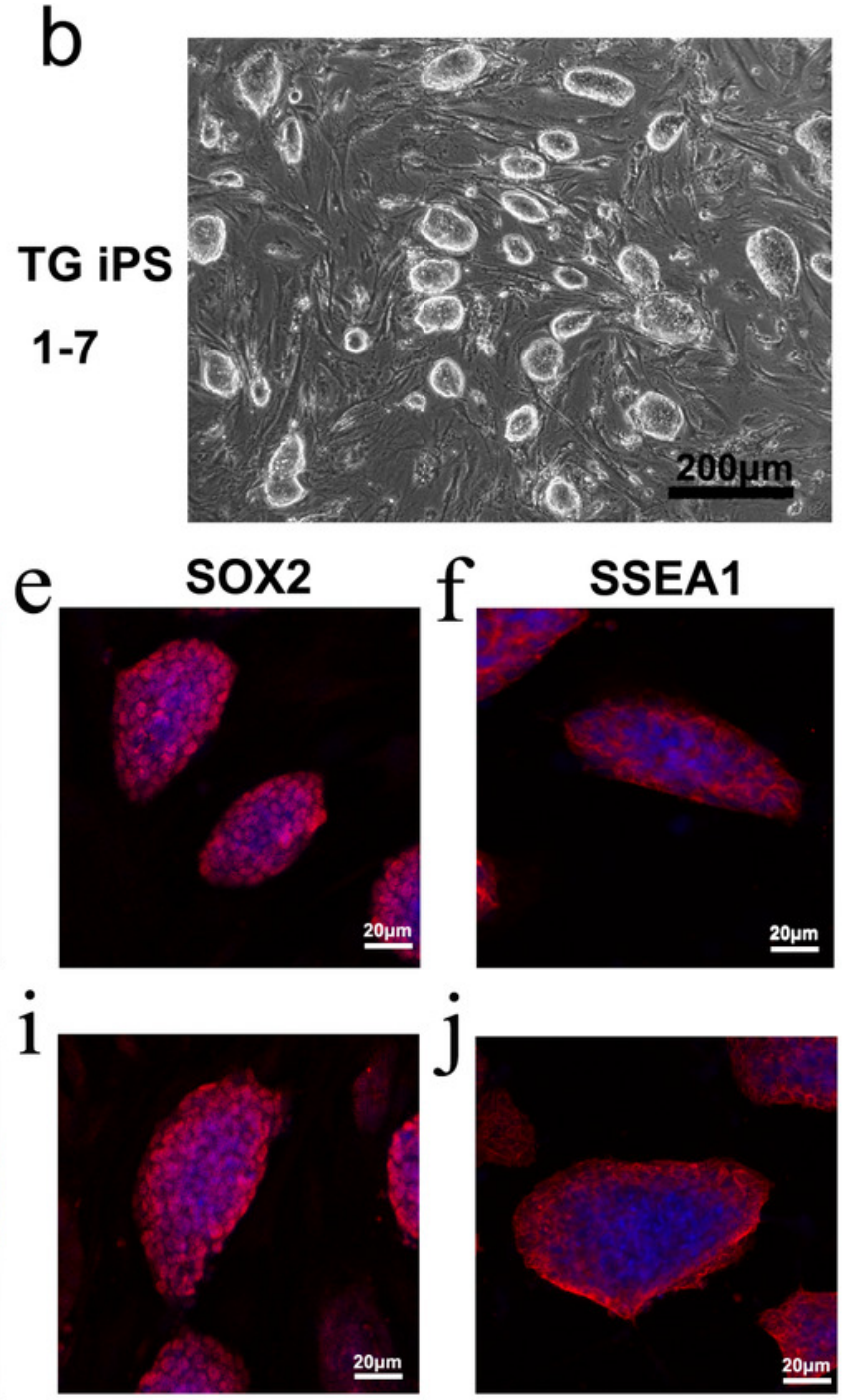
Figure 2

OCT4-MES, MES-FT, MES-ST, TG iPS 1-7, iPS-FT and iPS-ST express pluripotency genes.

Expression of pluripotency marker genes was evaluated by PCR, showing that OCT4-MES, MES-FT, MES-ST, TG iPS 1-7, iPS-FT and iPS-ST all express pluripotency marker genes, including Pou5f1, Sox2, Nanog, Rex1, Tbx3, Nr5a2, Utf1 and Lin28a.

*Note: Auto Gamma Correction was used for the image. This only affects the reviewing manuscript. See original source image if needed for review. 


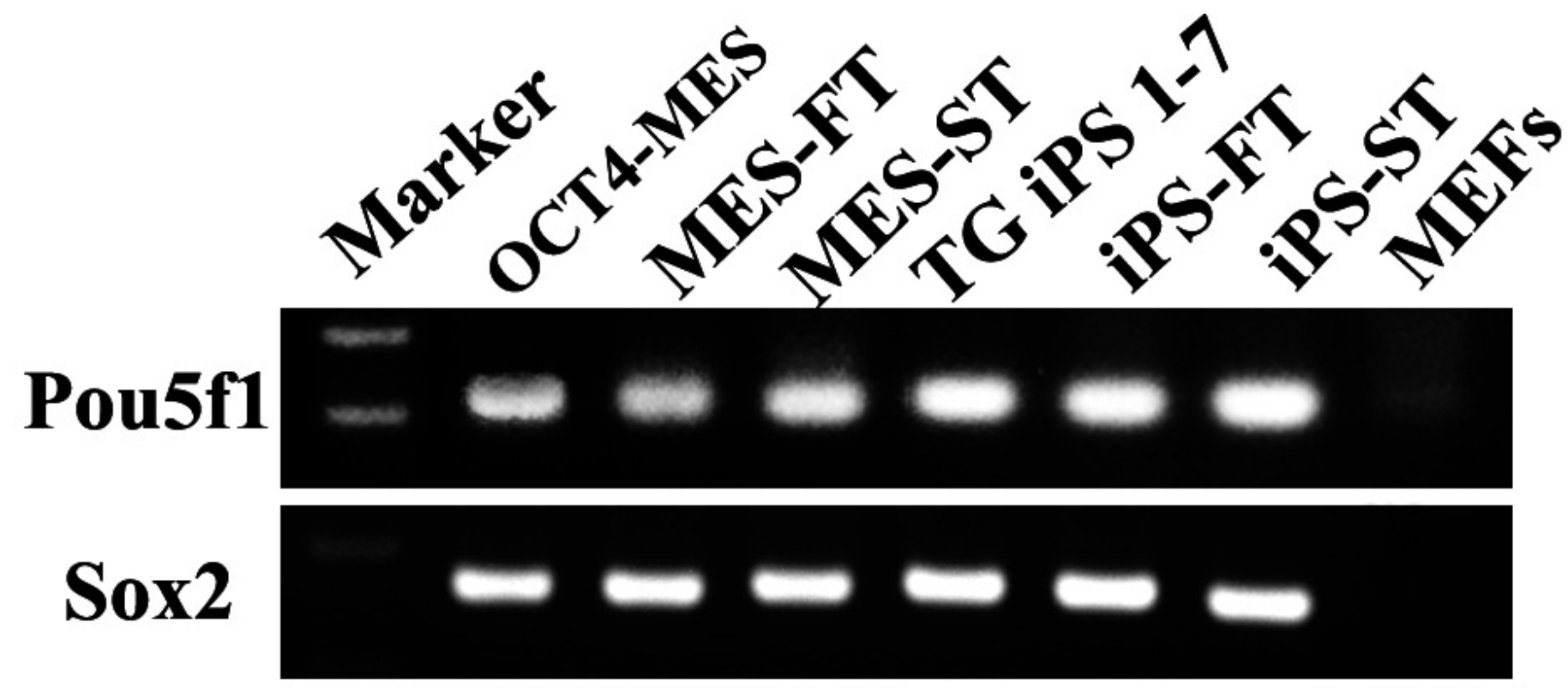

Nanog

$\operatorname{Rex1}$
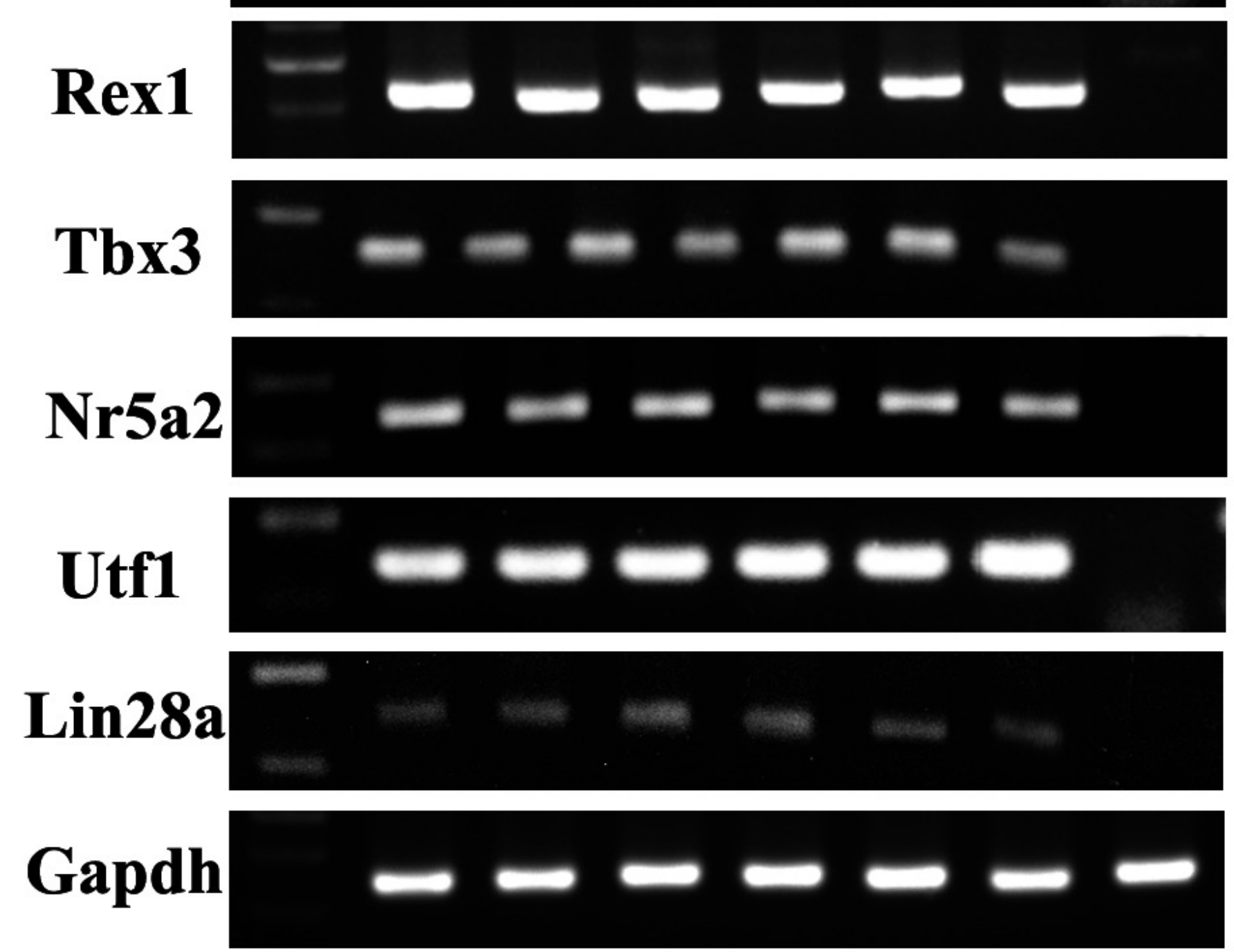


\section{Figure 3}

GFP-positive pluripotent cells are present in teratomas generated by OCT4-MES and TG iPS 1-7.

(a -f) Hematoxylin and eosin staining of teratomas derived from OCT4-MES (a-c) and TG IPS 1-7 (d-f). Products of all three germ layers are seen in the image: Ectoderm: epidermis with keratin ( $a$ and $d$ ). Mesoderm: smooth muscle ( $a$ and e). Endoderm: gastrointestinal lining cells/glands (c and f). Specified cells are indicated by arrows.

( $g$ and $\mathrm{h}$ ) Immunohistochemistry to detect the presence of GFP-positive pluripotent cells in teratomas generated by OCT4-MES (g) and TG IPS 1-7 (h). GFP-positive pluripotent cells were stained in brown with anti-GFP primary antibodies.

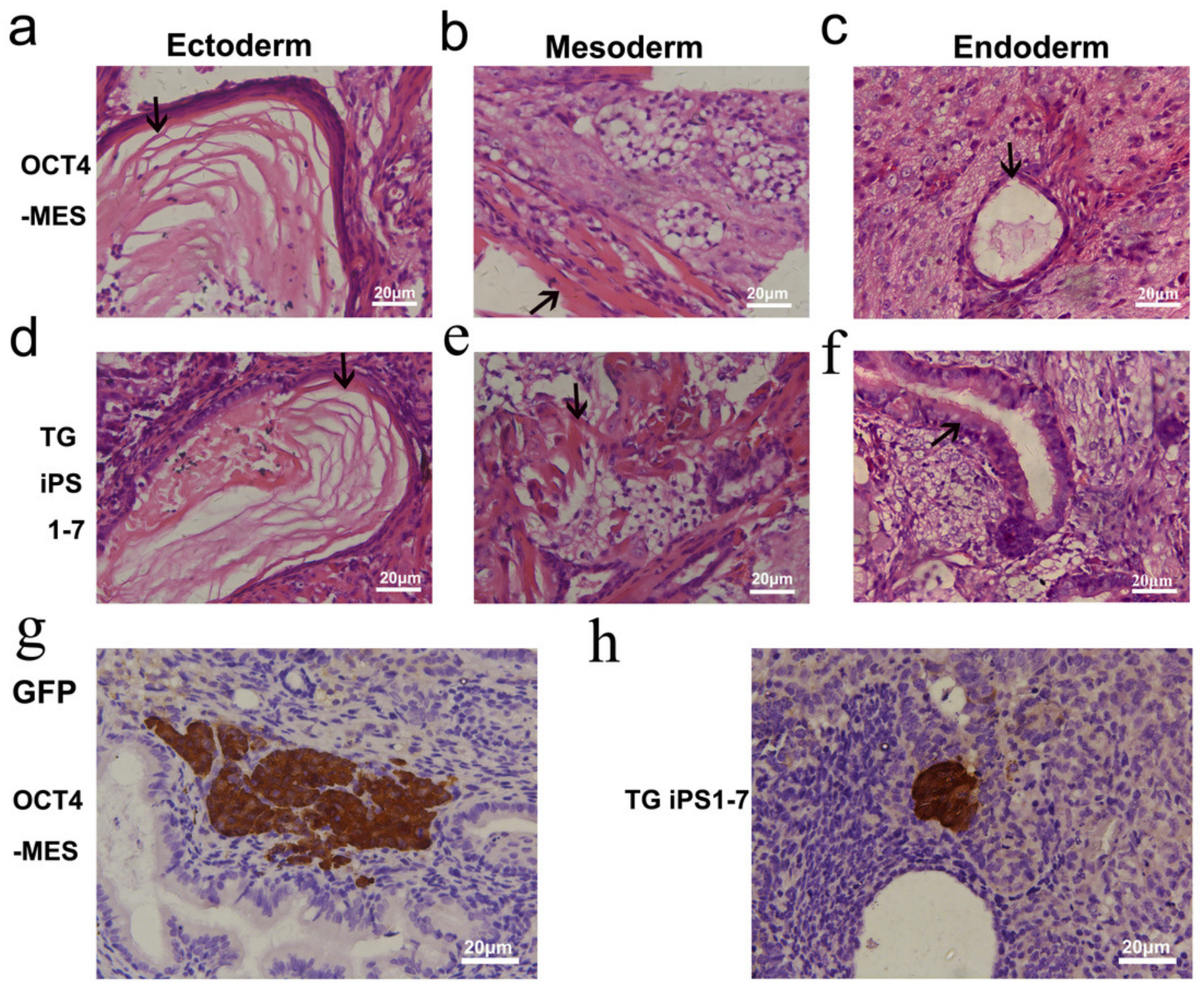




\section{Figure 4}

OCT4-positive pluripotent cells isolated from teratomas have typical mouse embryonic cell morphology .

( $a, b, d$ and e) A small number of OCT4-GFP positive cells were found among teratoma cells generated by OCT4-MES ( $a$ and $b$ ) and TG IPS 1-7 ( $d$ and f) cultured in MEF medium.

(c and f) MES-FT (c) and iPS-FT (f) have typical mouse embryonic cell morphology and are AP-positive when cultured in MES medium.

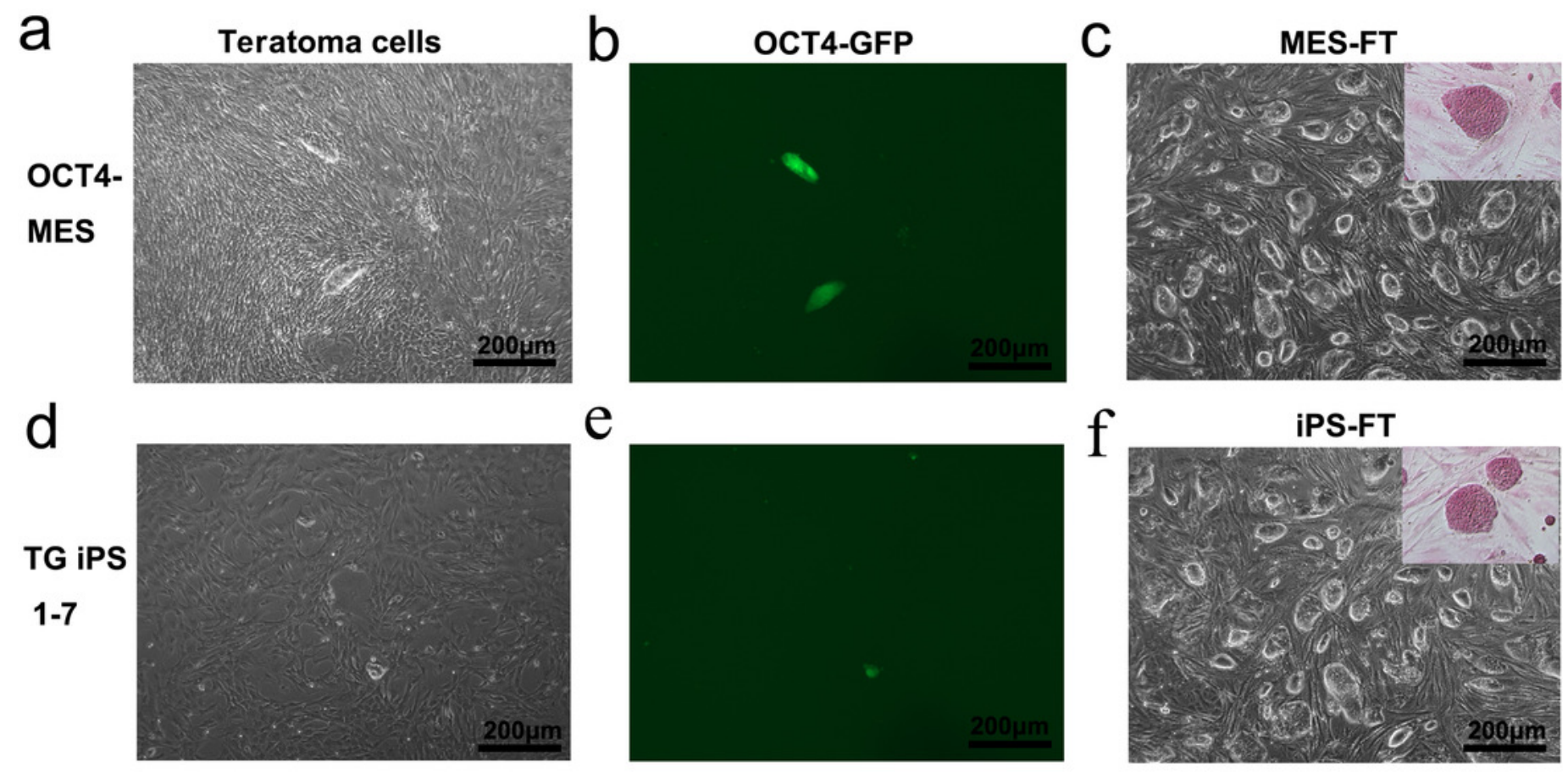




\section{Figure 5}

\section{MES-FT and iPS-FT own self-renewal and pluripotency ability.}

(a-h) Immunofluorescent staining of pluripotency markers OCT4, NANOG, SSEA1 and SOX2 in MES-FT (a-d) and iPS-FT (e-h). Both types of cells expressed all four markers.

(i-n) Hematoxylin and eosin staining of teratomas derived from MES-FT (i-k) and iPS-FT (I-n). Products of all three germ layers are seen in the image: Ectoderm: epidermis with keratin ( $i$ and $I)$. Mesoderm: smooth muscle ( $j$ and $m$ ). Endoderm: gastrointestinal lining cells/glands $(k$ and $n)$. Specified cells are indicated by arrows.

( 0 and p) Immunohistochemistry to detect the presence of GFP-positive pluripotent cells in teratomas generated by MES-FT (o) and iPS-FT (p). GFP-positive pluripotent cells were stained in brown with anti-GFP primary antibodies. 


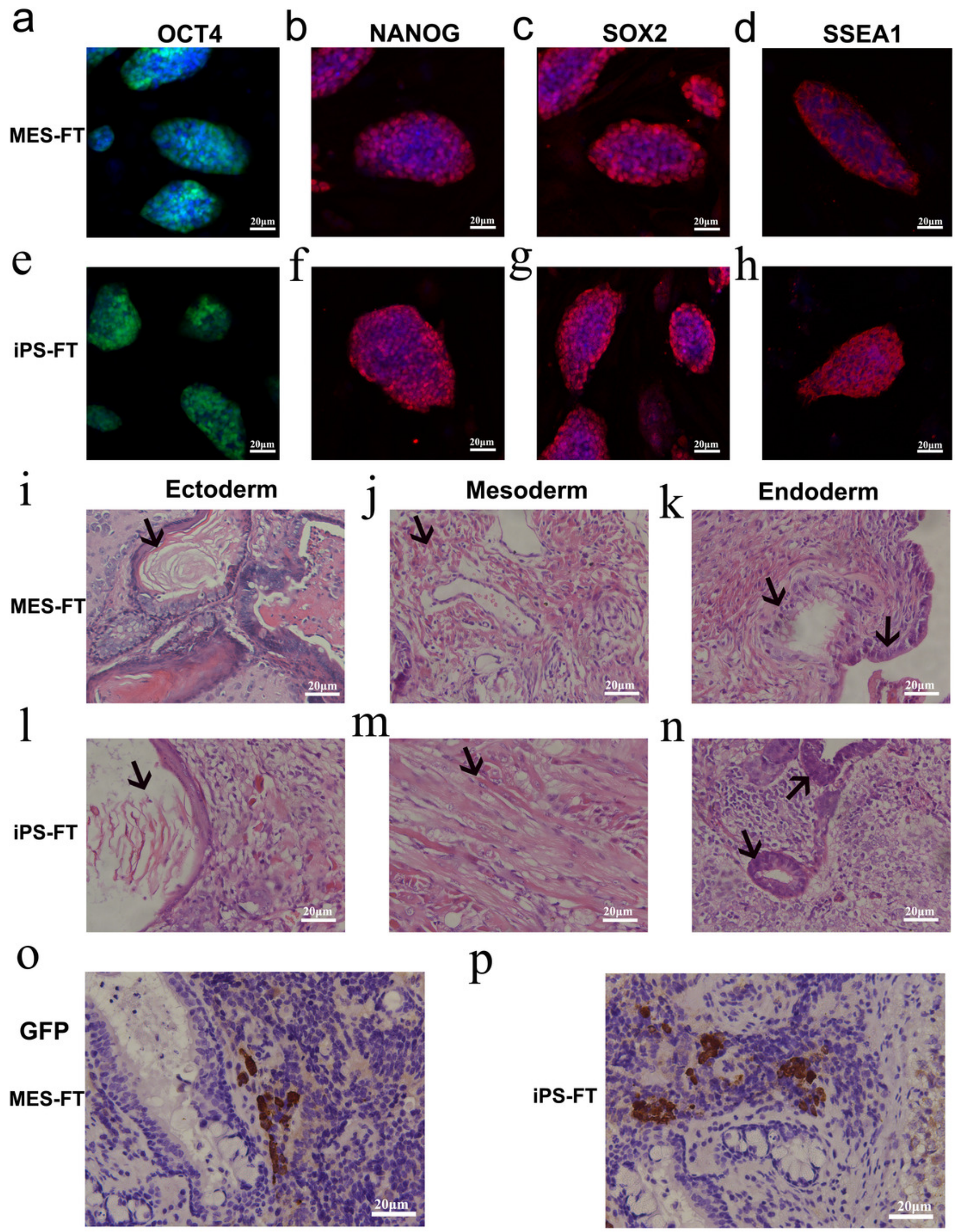




\section{Figure 6}

MES-ST and iPS-ST owned PSCs characteristic.

( $a, b, d$ and e) A small number GFP-positive cells were found among teratoma cells generated by MES-FT (a and $b$ ) and iPS-FT ( $d$ and e) cultured in MEF medium.

(c and f) MES-ST (c) and iPS-ST (f) have typical mouse embryonic cell morphology and are AP-positive when cultured in MES medium.

(g-k) Immunofluorescent staining of pluripotency markers OCT4, NANOG, SSEA1 and SOX2 in MES-ST (g-j) and iPS-ST $(k-n)$. Both MES-ST $(g-j)$ and iPS-ST $(k-n)$ expressed all four markers.

a

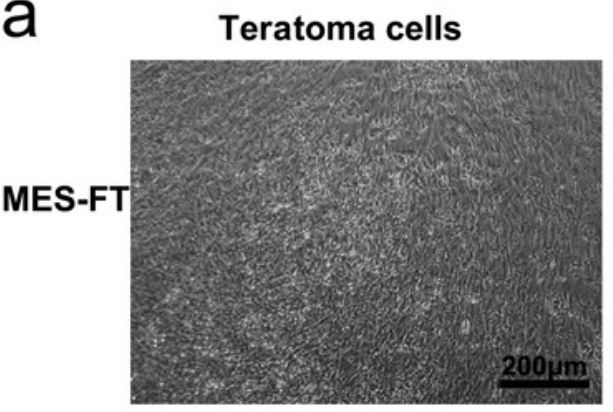

d

iPS-FT

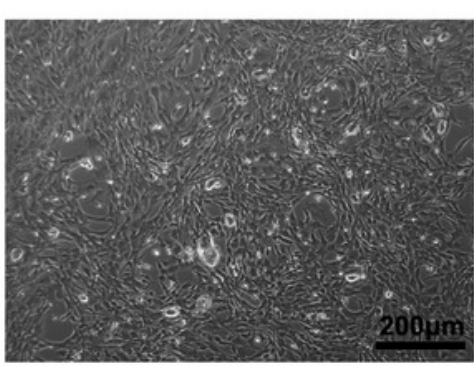

g

MES-ST

iPS-ST

h k

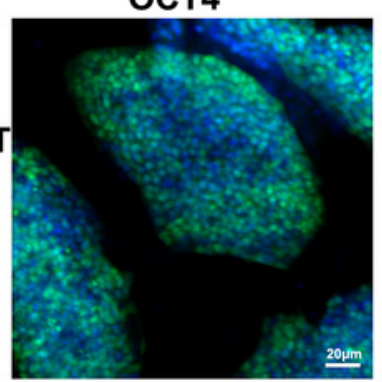

b

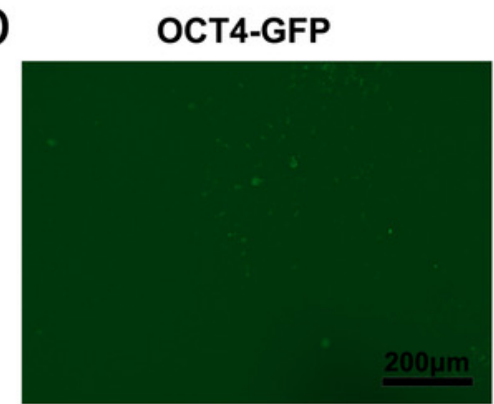

e

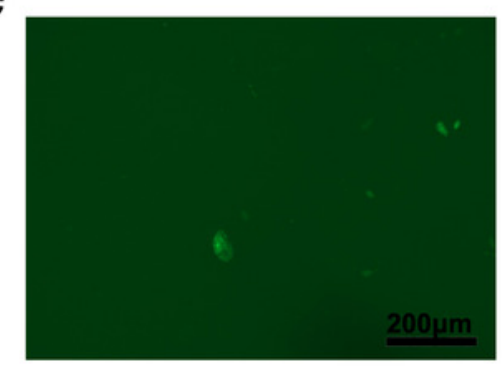

NANOG

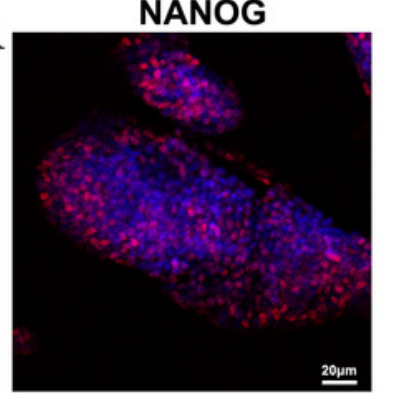

$\mathrm{i}$

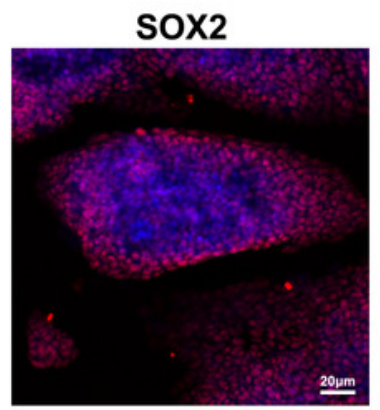

20um
C

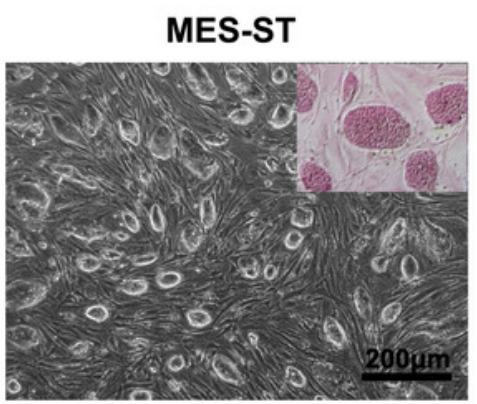

f

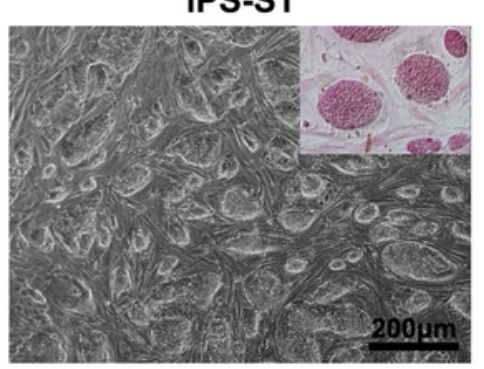

j SSEA1

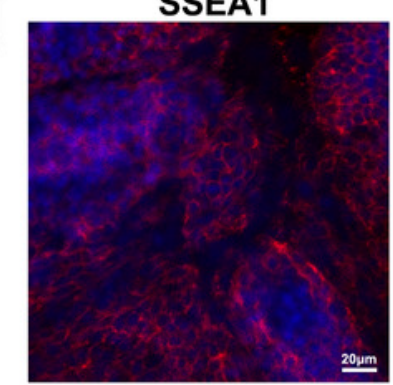

$\mathrm{m}$

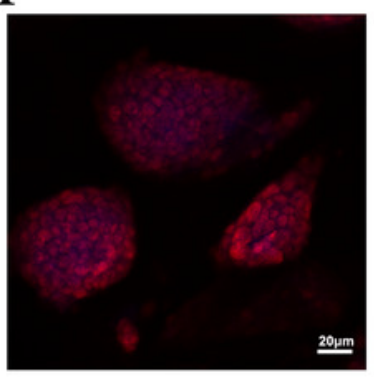

n

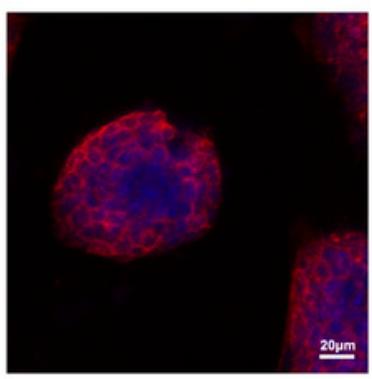




\section{Figure 7}

OCT4-MES, TG iPS 1-7, MES-FT, iPS-FT, MES-ST and iPS-ST express pluripotency genes; MES-FT, iPS-FT, MES-ST and iPS-ST more highly express several markers associated with germ cell formation.

(a) The expression levels of Pou5f1, Nanog and Lin28a in OCT4-MES, MES-FT and MES-ST were determined by qPCR. Both MES-FT and MES-ST highly expressed Lin28a, and MES-FT also highly expressed Pou5f1.

(b) The expression levels of pou5f1, Nanog and Lin28a in TG iPS 1-7, iPS-FT and iPS-ST were determined by qPCR. iPS-FT and iPS-ST highly expressed pou5f1 and Lin28a.

(c) The expression levels of Dazl, Stella, Stra8 and Vasa in Oct4-MES, MES-FT and MES-ST were determined by qPCR. Both MES-FT and MES-ST highly expressed Stra8. MES-ST also highly expressed Dazl and Stella.

(d) The expression levels of Dazl, Stella, Stra8 and Vasa in TG iPS 1-7, iPS-FT and iPS-ST were determined by qPCR. iPS-FT and iPS-ST highly expressed Dazl, Stra8 and Vasa. iPS-FT also highly expressed Stella.

Relative expression was quantified using the comparative threshold cycle (Ct) method ( $\left.2^{-\Delta c t}\right) . n=3$, Gapdh, EF $1 \alpha$ and $\beta$-tubulin were used as references. ${ }^{*} p<0.05$. 


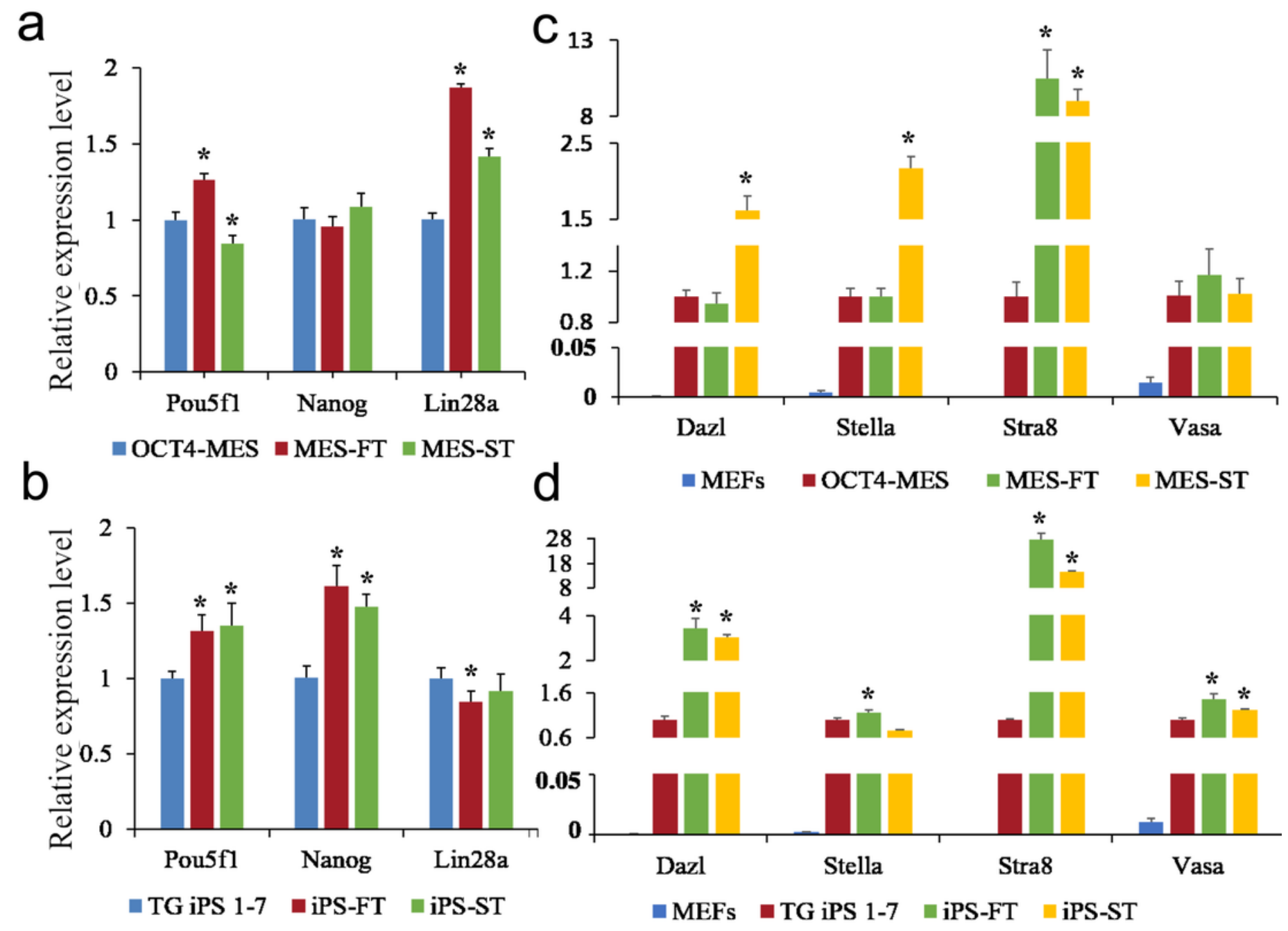

